Open Access

\title{
Low-FODMAP formula improves diarrhea and nutritional status in hospitalized patients receiving enteral nutrition: $a$ randomized, multicenter, double-blind clinical trial
}

So Ra Yoon', Jong Hwa Lee², Jae Hyang Lee', Ga Yoon Na², Kyun-Hee Lee ${ }^{3}$, Yoon-Bok Lee ${ }^{3}$, Gu-Hun Jung ${ }^{3}$ and Oh Yoen Kim ${ }^{\text {* }}$

\begin{abstract}
Background: Fermentable oligosaccharides, disaccharides, monosaccharides, and polyols (FODMAPs) are poorly absorbed, short-chain carbohydrates that play an important role in inducing functional gut symptoms. A low-FODMAP diet improves abdominal symptoms in patients with inflammatory bowel disease and irritable bowel syndrome. However, there were no study for the effect of FODMAP content on gastrointestinal intolerance and nutritional status in patients receiving enteral nutrition (EN).

Methods: In this randomized, multicenter, double-blind, 14-day clinical trial, eligible hospitalized patients receiving EN $(n=100)$ were randomly assigned to three groups; 84 patients completed the trial (low-FODMAP EN, $n=30$; moderate-FODMAP EN, $n=28$; high-FODMAP EN, $n=26$ ). Anthropometric and biochemical parameters were measured; stool assessment was performed using the King's Stool Chart and clinical definition.
\end{abstract}

Results: Baseline values were not significantly different among the three groups. After the 14-day intervention, diarrhea significantly improved in the low-FODMAP group than in the moderate- and high-FODMAP groups $(P<0.05)$. King's Stool scores in diarrhea subjects were significantly and steadily reduced in the low-FODMAP group compared with the other two groups ( $P$ for time and EN type interaction $<0.05$ ). BMl increased significantly in the low- and high-FODMAP groups during the intervention $(P<0.05$ for both), and showed a trend toward increasing in the moderate-FODMAP group $(P<0.10)$. Serum prealbumin increased significantly in all groups by 14 -day; by 3 -day, it had increased to the levels at 14-day in the low-FODMAP group. At 14-day, serum transferrin had increased significantly in the moderate-FODMAP group. In addition, subjects were classified by final condition (unimproved, normal maintenance, diarrhea only improved, constipation only improved, and recurrent diarrhea/constipation improved). Seventy-five percent of the diarrhea improved group consumed the low-FODMAP EN formula. 38.5 and $46.2 \%$ of recurrent diarrhea/constipation improved group consumed the low- and moderate-FODMAP EN respectively. BMI significantly increased in all groups except the unimproved. Prealbumin levels significantly increased in the diarrhea-improved and recurrent diarrhea/constipation groups at 3-day and continued by 14-day, and in the constipation-improved group at 14-day. Transferrin levels significantly increased in the diarrhea-improved and recurrent diarrhea/constipation groups at 14-day.

(Continued on next page)

\footnotetext{
* Correspondence: oykim@dau.ac.kr

${ }^{1}$ Department of Food Science Nutrition, Dong-A University, Brain Busan 21

Project, Busan 604-714, Republic of Korea

Full list of author information is available at the end of the article
} 
(Continued from previous page)

Conclusion: Low-FODMAP EN may improve diarrhea, leading to improved nutritional status and facilitating prompt recovery from illness.

Keywords: Poorly absorbed, Short-chain carbohydrates, Enteral nutrition, Diarrhea, Prealbumin, Transferrin, body mass index

\section{Background}

Enteral nutrition (EN) is a common method of nutrition support for hospitalized patients with intact gastrointestinal function who are unable to eat or whose nutritional requirements are not satisfied with an oral diet [1]. Despite the benefits of EN (e.g., shortening the length of hospital stays and lowering rates of infectious complications, readmissions, and mortality) [2-4], gastrointestinal symptoms, such as diarrhea, constipation, bloating, abdominal pain, flatulence, and vomiting/nausea, frequently occur [5]. These symptoms may cause malnutrition (i.e., imbalanced fluid and electrolytes and/or inadequate intake/ absorption of nutrients), thereby preventing prompt recovery from illness $[6,7]$. Diarrhea, in particular, is a frequently observed gastrointestinal symptom in patients receiving EN [8-10]: it occurs in anywhere of hospitalized EN patients, depending on their health [11-13]. The cause of EN-associated diarrhea is unclear, but likely multifactorial. Absorption problems, feeding temperature, feeding method, and the high osmolality and nutrient composition of EN formulas are probable factors [14-19]. Many have endeavored to solve the problem by changing feeding method, temperature, or formula content [6], but the results have been inconsistent.

Recently, it has been suggested that diarrhea is associated with fermentable oligosaccharides, disaccharides, monosaccharides, and polyols (FODMAPs) which is poorly absorbed, short-chain carbohydrates [8, 9, 20-23]. FODMAPs are found in a wide variety of foods, including apples, mangos, and fructose syrup (fructose); onions, garlic, and rye (fructans); milk (lactose); legumes (galactans); and mushrooms, stone fruit, and some artificial sweeteners (polyols) [24]. They are poorly absorbed in the small intestine and, when delivered to the colon, may produce gas and subsequently cause luminal distension, disturb gut motility, and result in diarrhea [23-25]. A low-FODMAP diet significantly reduces gastrointestinal symptoms in patients with irritable bowel syndrome (IBS) and inflammatory bowel disease (IBD) [20-23, 26]. Of note, lowFODMAP dietary interventions improve gastrointestinal symptoms more in IBS patients with fructose malabsorption problems than in those without [22]. A retrospective EN study reported that the rate of diarrhea development was lower in patients given a lower-FODMAP formula than those given other types of formulas [8]: although length of hospital stay and duration of EN independently predicted diarrhea development, this study suggested that being provided with a lower-FODMAP formula could potentially mitigate these risks $[8,9]$.

To date, all the intervention studies for the association between gastrointestinal symptom and FODMAPs were performed in the patients with IBS or IBD, however there has been no prospective intervention study investigating the effect of FODMAP content on diarrhea and nutritional status in patients receiving EN. Therefore, this randomized, double-blind 14-day clinical trial investigated the effect of FODMAP content in EN formula on gastrointestinal intolerance and nutritional status in hospitalized patients receiving EN.

\section{Materials and methods}

\section{Study design and participants}

This study was a randomized, multicenter, double-blind, 14-day clinical trial. Between November 2013 and November 2014, participants were recruited from 10 hospitals located in Busan and its environs, in the Republic of Korea. Five were university hospitals (Dong-A University Hospital, Busan National University Hospital and Dong-Eui Medical Center in Busan, and Busan National University Hospital in Yangsan) and six were rehabilitation and long-term care hospitals (Bumin Hospital, Centum Erooda Hospital, Happy Medical Center, Inchang Hospital, Keunsol Medical Hospital, and Medwill hospital in Busan). All participants aged 20 years or over were hospitalized and receiving EN. Exclusion criteria included the following: 1) EN forbidden due to ileus, gastrectomy, bleeding in the digestive tract, etc.; 2) renal or liver dysfunction, e.g., renal failure, nephritis, nephrotic syndrome, hepatitis, cirrhosis, serum creatinine $>1.5 \mathrm{mg} / \mathrm{dl}$, serum urea nitrogen $>25 \mathrm{mg} / \mathrm{ml}$, serum aspartate aminotransferase $>40 \mathrm{U} / \mathrm{L}$, or serum alanine aminotransferase $>40 \mathrm{U} / \mathrm{L}$; 3) uncontrollable diabetes; and 4) pregnancy or breast-feeding. All study participants (or their legal guardians) were provided with detailed information about the study and provided written informed consent. One hundred sixty five subjects were assessed for eligibility. One-hundred eligible subjects were randomly assigned to low-FODMAP EN, moderate-FODMAP EN, or high-FODMAP EN for 14 days using a block randomization method. The 
patients, clinicians and all study personnel were blinded to the contents of the experimental EN formulas. The experimental EN formulas were indistinguishable color and provided in identical cans. Allocation concealment was maintained using a centralized, web-based randomization schedule accessible $24 \mathrm{~h}$ a day. Energy needs were estimated by a registered dietitian (RD) based on the patient's height, weight and disease condition. Basically, patients received intermittent nasogastric EN feeding (100-400 ml/20-40 min, 3 times/day). To avoid unexpected gastrointestinal problems due to a sudden change in EN formula, each patient basically had a 3-day adaptation period in which one-third the volume of the former formula was replaced with the new, experimental EN formula each day. Sixteen participants dropped out during the intervention period, leaving 84 to complete the study. Of the 16 dropouts, 2 were transferred to another hospital, 2 stopped EN and changed to a transitional diet, 4 severe diarrheas and 8 withdrew for personal reasons. Finally, 84 patients completed the trial (low-FODMAP EN, $n=30$; moderate-FODMAP EN, $n=$ 28; high-FODMAP EN, $n=26$ ) were included for analysis. The study protocol was approved by the Institutional Review Board of Dong-A University (the representative, approval no. 2-1040709-AB-N-01201310-BR-03-03) and carried out in accordance with the Helsinki Declaration. The study's clinicaltrial.gov identifier is NCT02353689.

\section{Nutrient composition of experimental enteral nutrition (EN) formulas}

Three types of EN formula were provided by Dr. Chung's Foods Co., Ltd. (Cheongju, Chungbuk, Republic of Korea); the FODMAP content and nutrient composition of each are described in Table 1. All the experimental EN formulas contained the same number of calories per can $(200 \mathrm{kcal} / 200 \mathrm{ml})$, but different FODMAP contents (low-FODMAP: $0.320 \mathrm{~g} / \mathrm{can}$; moderate-FODMAP: $0.753 \mathrm{~g} / \mathrm{can}$; high-FODMAP: $1.222 \mathrm{~g} / \mathrm{can}$ ). Most of nutrient contents except some micronutrients are similar in all the EN formulas and they were formulated following to and within the guideline of Korean dietary reference intakes.

\section{Data collection}

Baseline data included: patient demographics (age, gender, height and body weight), disease history, dietitian assessment of nutritional requirement etc. Data were collected daily for up to 14 days post-randomization for stool assessment, energy intake, time taken to reach $100 \%$ experimental EN feeding. Anthropometric and biochemical parameters were also collected at 0-day (before starting), 3-day ( $4^{\text {th }}$ day morning) and 14-day (after the intervention) for the monitoring of nutritional status.

\section{Outcomes}

The primary outcome was the improvement of diarrhea assessed by the King's Stool chart and the clinical definition. Secondary outcomes were the levels of plasma prealbumin and transferrin, the markers for short-term nutritional status. Other outcomes were changes of anthropometric parameters, other biochemical parameters and other gastrointestinal symptoms.

\section{Stool assessment}

Stool assessment data were collected daily for up to 14 days. It was performed using the King's Stool Chart, which incorporates descriptors of stool frequency, weight, and consistency, as previously described [27-29]. The chart has been validated for use in free-living individuals, patients at high-risk of diarrhea and patients receiving enteral nutrition [27.28]. Briefly, the chart comprises three categories of stool weight ( $<100 \mathrm{~g}, 100-200 \mathrm{~g},>200 \mathrm{~g})$ and four categories of stool consistency (hard and formed, soft and formed, loose and unformed, liquid). This results in 12 possible combinations of stool weight and consistency, each accompanied by a verbal and pictorial descriptor to assist accurate characterization [27-29]. Each category is also assigned an alphabetical code, so that physicians, nurses, and dieticians can record and communicate fecal output using standard verbal and pictorial descriptors. Fecal frequency is incorporated by recording the code of each feces passed; each category is assigned a score, enabling the summation of all scores in a 24-h period to obtain a daily fecal score. In this study, diarrhea was classified as a daily fecal score of 15 or more. Diarrhea was also classified using a clinical definition: 1) unformed or liquid stools for two or more days; or 2) unformed and liquid stools $(\geq 200 \mathrm{~g})$ three times or more per day. Constipation was defined as a condition of fewer than three bowel movements per week or no defecation for three or more days, requiring stool softeners or an enema at least once.

\section{Anthropometric parameters, blood pressure, and blood collection}

Height and body weight were measured when possible (62 of 84 patients). Body mass index (BMI) was calculated as body weight divided by height squared $(\mathrm{kg} /$ $\mathrm{m}^{2}$ ). Blood pressure (BP) was obtained from the left arm of seated individuals with an automatic BP monitor (HEM-7220, OMRON, Matsusaka, Japan) after a short rest. Triceps skin fold thickness (TSF, $\mathrm{cm}$ ), midarm circumference (MAC, $\mathrm{mm}$ ) and midarm muscle circumference $($ MAMC $[\mathrm{cm}]=$ TSF $[\mathrm{cm}]-$ MAC $[\mathrm{cm}] \times 3.14$ ) were also measured. Blood specimens were collected three times over the intervention 
Table 1 FODMAP content and nutrient composition of experimental EN formulas

\begin{tabular}{|c|c|c|c|}
\hline Per can (200 mL) & Low-FODMAP & Moderate-FODMAP & High-FODMAP \\
\hline Calorie (kcal) & 200 & 200 & 200 \\
\hline Total FODMAPs (g) & 0.320 & 0.753 & 1.222 \\
\hline Fructose (g) & - (N.D.) & - & - \\
\hline Lactose $(\mathrm{g})$ & - & - & - \\
\hline Raffinose (g) & 0.079 & 0.229 & 0.285 \\
\hline Stachyose (g) & - & - & 0.239 \\
\hline 1-Kestose (g) & - & - & 0.511 \\
\hline Nystose (g) & - & - & - \\
\hline 1-Fructofuranosylnystose (g) & 0.233 & 0.509 & 0.411 \\
\hline Carbohydrates (g) & 28 & 32 & 31 \\
\hline Sugar (g) & 0 & 1 & 2 \\
\hline Fiber (g) & 3 & 3 & 4.3 \\
\hline Protein (g) & 9 & 8 & 9 \\
\hline Fat (g) & 7 & 6 & 6 \\
\hline Sodium (mg) & 135 & 180 & 135 \\
\hline Vitamin A ( $\mu g R E)$ & 225 & 140 & 150 \\
\hline Vitamin B1 (mg) & 0.24 & 0.3 & 0.24 \\
\hline Vitamin B2 (mg) & 0.3 & 0.36 & 0.3 \\
\hline Vitamin B6 (mg) & 0.3 & 0.45 & 0.3 \\
\hline Vitamin B12 $(\mu \mathrm{g})$ & 0.96 & 1 & 0.48 \\
\hline Vitamin C (mg) & 40 & 30 & 20 \\
\hline Vitamin D $(\mu \mathrm{g})$ & 1 & 1 & 1 \\
\hline Vitamin E (mg a-TE) & 4.8 & 5 & 2 \\
\hline Vitamin K $(\mu \mathrm{g})$ & 15 & 11 & 15 \\
\hline Folic acid $(\mu \mathrm{g})$ & 80 & 75 & 80 \\
\hline Niacin (mg NE) & 3.2 & 2.6 & 3.2 \\
\hline Biotin $(\mu \mathrm{g})$ & 6 & 6 & 6 \\
\hline Pantothenic acid (mg) & 1 & 1 & 1 \\
\hline Calcium (mg) & 150 & 140 & 140 \\
\hline Phosphorus (mg) & 140 & 140 & 140 \\
\hline Magnesium (mg) & 58 & 22 & 58 \\
\hline Zinc (mg) & 4 & 2.4 & 2 \\
\hline Iron (mg) & 2 & 2.3 & 2 \\
\hline Potassium (mg) & 225 & 308.5 & 220 \\
\hline Manganese (mg) & 0.8 & 0.4 & 0.7 \\
\hline Copper (mg) & 0.16 & 0.23 & 0.16 \\
\hline lodine (mg) & 30 & 23 & 30 \\
\hline
\end{tabular}

N.D. non-detected, NE niacin equivalents, $a$-TE a-tocopherol

period (0-day, 3-day and 14-day). After an overnight fast, venous blood specimens were collected in EDTA-treated or plain tubes. The tubes were immediately placed on ice until they arrived at the analytical laboratory $(1-3 \mathrm{~h})$. Blood specimens were then separated into plasma and serum and stored at $-80{ }^{\circ} \mathrm{C}$ until analysis.
Serum glucose, lipid profile, albumin, prealbumin, transferrin and blood cell counts

Fasting glucose was measured using a glucose oxidase method (Glucose Analyzer Beckman Instruments, Irvine, CA, USA), as described in a previous report [30]. Serum total cholesterol, triglycerides, and low-density lipoprotein (LDL) cholesterol levels were measured using 
commercially available kits on a Hitachi 7150 Autoanalyzer (Hitachi Ltd., Tokyo, Japan), as described previously [31]. After precipitating serum chylomicrons, LDL-cholesterol, and very low-density lipoprotein with dextran sulfate-magnesium, the high-density lipoprotein (HDL) cholesterol left in the supernatant was measured using a previously described enzymatic method [31]. Serum fasting albumin, preabumin and transferrin were measured using commercially available kits on a Hitachi 7150 Auto-analyzer (Hitachi Ltd., Tokyo, Japan). White blood cell (WBC) and absolute lymphocyte (ALC) counts were determined using the HORIBA ABX diagnostics system (HORIBA ABX SAS, Parc Euromedicine, France).

\section{Safety parameters: liver and kidney functions}

Serum aspartate aminotransferase (AST) and alanine aminotransferase (ALT) were measured using a modified International Federation of Clinical Chemistry (IFCC) UV method. Serum creatinine was measured with a kinetic colorimetric (Jaffe) assay. Serum concentrations of blood urea nitrogen (BUN) and uric acid were measured using a kinetic UV Assay.

\section{Serum high-sensitivity C-reactive protein and plasma interleukin-6, interleukin-1 $\beta$, and tumor necrosis factor- $\alpha$} Serum high-sensitivity C-reactive protein (hs-CRP) was measured with an ADVIA 1650 system (Bayer, Tarrytown, NY, USA) using a commercially available, high-sensitivity CRP-Latex(II) X2 kit (Seiken Laboratories Ltd., Tokyo, Japan), which allows detection of CRP in the $0.001-31 \mathrm{mg} / \mathrm{dL}$ range, as described in a previous report [31]. Plasma concentrations of interleukin-6 (IL-6), IL-1 $\beta$, and tumor necrosis factor$\alpha$ (TNF- $\alpha)$ were measured using the Quantikine ${ }^{\circ}$ HS ELISA Kit (R\&D systems, Inc., Minneapolis, MN, USA), according to the manufacturer's instructions. The resulting color reaction was measured using the iMark $^{\mathrm{m}}$ microplate absorbance reader (Bio-Rad Laboratories, Hercules, CA, USA). The wavelength correction was set to 490 and $560 \mathrm{~nm}$.

\section{Statistical analysis}

Statistical analyses were performed with Win SPSS v21.0 (SPSS Inc., Chicago, IL). Sample-size calculation was based on the primary outcome, improvement of diarrhea during the 14-day intervention period. A sample size of 25 subjects per group with a significance level of 0.05 yielded $95 \%$ power, therefore 100 participants were enrolled to allow for over an estimated $70 \%$ retention rate across the study period. No stopping rules or interim analyses were planned. Differences in continuous variables between the groups were assessed using one-way analysis of variance (ANOVA) followed by the
Bonferroni correction. Differences in continuous variables within a group, before and after the intervention, were analyzed using paired $t$-tests. Repeated measure ANOVA with the Bonferroni correction was also performed to examine the interactive effect between time and EN type (Time $x$ EN type effect) on the King's Stool scores change (\%). Differences in non-continuous variables were analyzed with the chi-square test. Skewed variables were log transformed prior to statistical analysis. However, for descriptive purposes, means $\pm \mathrm{SE}$ are presented using untransformed values. A twotailed $P$-value $<0.05$ was considered statistically significant.

\section{Results}

\section{Baseline characteristics of study participants}

Table 2 presents baseline characteristics of the study participants completed. There were no significant differences in age, height, body weight, BMI, MAC, TSF, MAMC, BP, sex, disease history, energy intake goals, average energy intake during the intervention, or time taken to reach $100 \%$ experimental EN feeding among the three groups.

\section{Distribution of major gastrointestinal symptoms before and after the intervention}

There were no significant differences in the baseline distribution of gastrointestinal symptoms [normal, diarrhea, constipation, or recurrent diarrhea/constipation $(D / C)]$ among the three EN treatment groups (Additional file 1: Table S1). 'diarrhea"' or 'constipation"' include recurrent $\mathrm{D} / \mathrm{C}$ before the intervention, 'diarrhea only' indicates subjects who suffered from only diarrhea without recurrent $\mathrm{D} / \mathrm{C}$ before the intervention, 'constipation only' indicates subjects who suffered from only constipation without recurrent $\mathrm{D} / \mathrm{C}$ before the intervention. Gastrointestinal symptoms were daily observed for the 14 intervention days.

Figure 1 shows improvement (\%) of diarrhea according to EN types during the intervention. Each subject group was also subdivided into two part 'medication user included' and 'medication user excluded'. Diarrhea in the low-FODMAP group had improved significantly more than that in the moderate- or high-FODMAP groups after the intervention (final improvement). This pattern was similarly observed in each diarrhea subgroup Diarrhea improvements in the low-FODMAP group among subjects with diarrhea* including or excluding medication users were $73.3 \%(P=0.046)$ and $71.4 \%(P=0.028)$, respectively, among those with diarrhea only including or excluding medication users were $60.0 \%(P=0.047)$ and $60.0 \%$ $(P=0.035)$, respectively. Interestingly, diarrhea improvement among the subjects with diarrhea only, but no medication users was observed only in the low-FODMAP 
Table 2 Baseline characteristics of study subjects

\begin{tabular}{|c|c|c|c|}
\hline & Low-FODMAP $(n=30)$ & Moderate-FODMAP $(n=28)$ & High-FODMAP $(n=26)$ \\
\hline Age (years) & $60.1 \pm 2.92$ & $60.6 \pm 3.03$ & $62.5 \pm 2.70$ \\
\hline Female (n, \%) & $9(30.0)$ & $9(32.1)$ & $8(30.8)$ \\
\hline Weight (kg) & $55.4 \pm 2.37$ & $56.7 \pm 2.61$ & $55.9 \pm 2.48$ \\
\hline BMI $\left(\mathrm{kg} / \mathrm{m}^{2}\right)^{\mathrm{a}}$ & $19.9 \pm 0.68$ & $20.6 \pm 0.77$ & $20.4 \pm 0.75$ \\
\hline MAC (cm) & $25.2 \pm 0.53$ & $25.4 \pm 0.70$ & $24.2 \pm 0.80$ \\
\hline TSF $(\mathrm{mm})$ & $11.0 \pm 0.89$ & $12.2 \pm 1.04$ & $10.1 \pm 0.88$ \\
\hline MAMC (cm) & $21.8 \pm 0.50$ & $21.5 \pm 0.53$ & $21.0 \pm 0.72$ \\
\hline Systolic BP (mmHg) & $111.2 \pm 2.85$ & $116.3 \pm 2.21$ & $116.5 \pm 2.76$ \\
\hline Diastolic BP (mmHg) & $74.2 \pm 2.52$ & $73.5 \pm 1.52$ & $75.0 \pm 1.89$ \\
\hline \multicolumn{4}{|l|}{ Disease history } \\
\hline Hypertension & $13(43.3 \%)$ & $16(57.1 \%)$ & $12(46.2 \%)$ \\
\hline Diabetes $^{b}$ & $5(16.7 \%)$ & $5(17.9 \%)$ & $6(23.1 \%)$ \\
\hline Stroke & $23(76.7 \%)$ & $15(53.6 \%)$ & $18(69.2 \%)$ \\
\hline Cardiovascular disease & $4(13.3 \%)$ & $3(10.7 \%)$ & $6(23.1 \%)$ \\
\hline Hyperlipidemia & $3(10.0 \%)$ & $7(25.0 \%)$ & $2(8.00 \%)$ \\
\hline Cancer & $1(3.30 \%)$ & $1(3.60 \%)$ & $2(8.00 \%)$ \\
\hline Energy intake goal (kcal/day) & $1273.3 \pm 32.5$ & $1310.7 \pm 45.5$ & $1269.2 \pm 46.7$ \\
\hline Average energy intake (kcal/day) & $1267.2 \pm 33.8$ & $1302.6 \pm 44.5$ & $1270.5 \pm 47.0$ \\
\hline Time to reach $100 \%$ experimental EN feeding (days) & $2.57 \pm 0.22$ & $2.57 \pm 0.29$ & $2.58 \pm 0.21$ \\
\hline
\end{tabular}

$B M I$ body mass index, $B P$ blood pressure, MAC midarm circumference, MAMC midarm muscle circumference, TSF triceps skin fold thickness Means \pm SE or $\mathrm{n}(\%)$

${ }^{a} n=62$

${ }^{b}$ Well-controlled diabetes

There were no significant differences in the baseline values among the three groups

group. Within the seven days from the start of intervention (for early improvement), diarrhea showed a trend towards improvement in the low-FODMAP group compared with the moderate- and high-FODMAP groups, but the difference did not reach statistical significance (Fig. 1).

Constipation improvements were also observed after the intervention but not significantly different among the three EN treatment groups (Additional file 1: Figure S1.A). This pattern was similarly observed in each constipation subgroup. Although constipation improvements seemed slightly higher in the highFODMAP group than the other EN groups, the differences did not reach the statistical significances, which might be related with the small number of subjects in these groups. As shown in Additional file 1: Figure S1.B, diarrhea had improved greater in the lowFODMAP group than the moderate- or highFODMAP groups among the subjects with recurrent $\mathrm{D} / \mathrm{C}$. This pattern was observed in each subject group including or excluding medication users (in the lowFODMAP group: $80 \%, P=0.017 ; 100 \% P=0.030$, respectively). Constipation had improved only in the high-FODMAP group ( $22.2 \%$ of 'the medication user included'; $33.3 \%$ of 'the medication user excluded'), but which did not reach the statistical significance. Both diarrhea and constipation were slightly improved in the low- (20\% of 'the medication user included'), moderate- ( $40 \%$ of 'medication user included'; $33.3 \%$ of 'the medication user excluded') and high-FODMAP (11.1 \% of 'medication user included') groups, but which were not significantly different among the groups. Participants with normal condition at baseline maintained this condition, except for one case occurrence of constipation in the moderate-FODMAP group. A few other gastrointestinal symptoms (e.g., mild abdominal pain or bloating) were observed during the intervention, but their incidence was neither high nor significantly different among the three groups $(10.0 \%$ in the low-FODMAP group, $10.7 \%$ in the moderate-FODMAP group, and $30.8 \%$ in the high-FODMAP group).

\section{Changes in King's Stool score during intervention}

Data for stool assessment using the King's Stool scores chart were collected daily during the intervention period. For graphical presentation, the observation periods were categorized into four time windows: the adaptation period (D1-3, first three days: 0-day, 1-day, 2-day), the early period (D4-6: 3-day, 4-day, 5- 


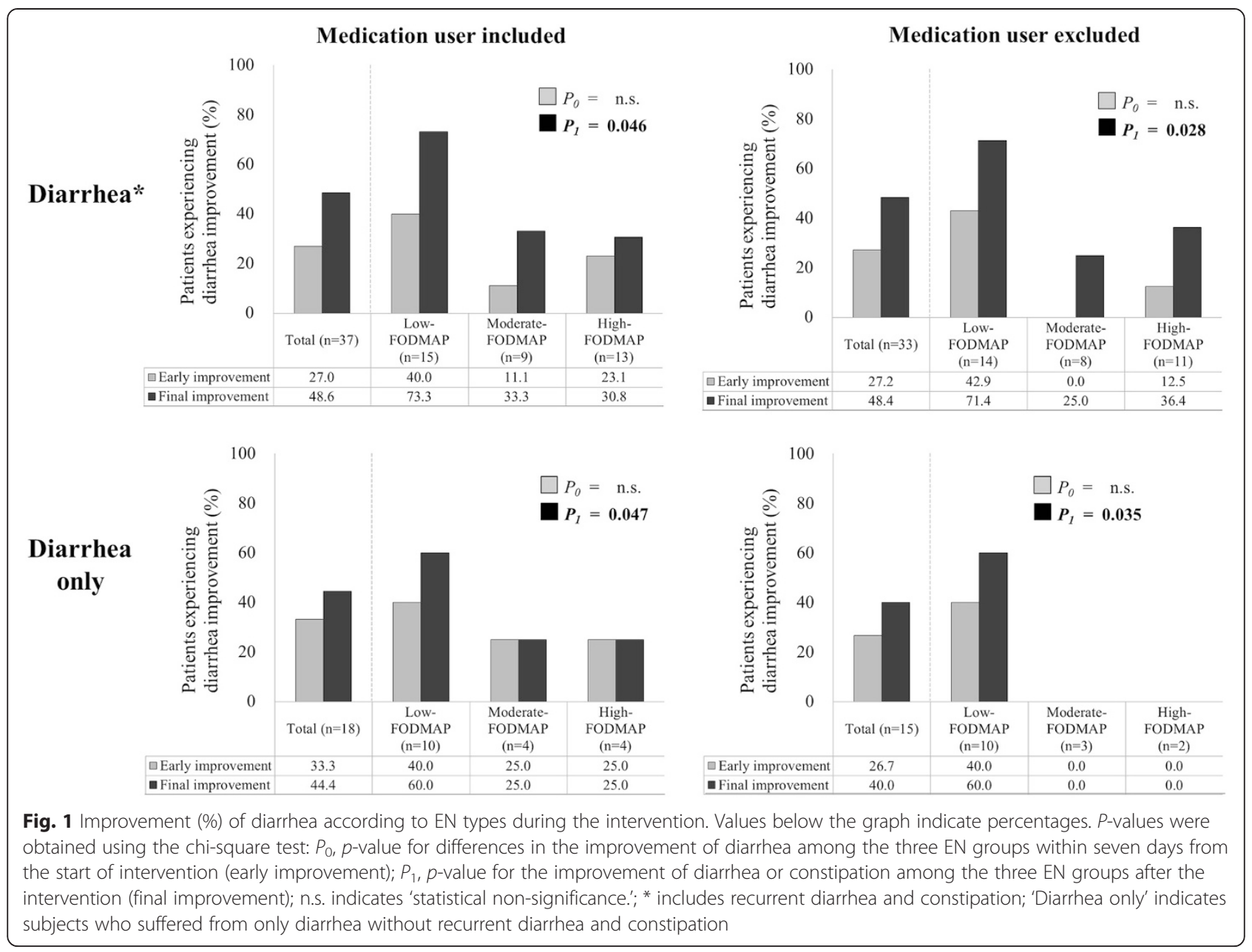

day), the mid period (D7-9: 6-day, 7-day, 8-day), and the late period (D12-14, last three days: 11-day, 12day, 13-day). The mean scores in each observation period were the average values calculated from the scores in each day during that period. Figure 2 presents \% change of King's Stool scores from the adaptation period (D1-3) to the early period (D4-6), the mid period (D7-9) and late period (D12-14) in the diarrhea subjects (Diarrhea* and 'Diarrhea only' groups). We also subdivided each subject group into two part 'medication user included' and 'medication user excluded'. Repeated Measure ANOVA test was performed to examine the interactive effect between time and EN type (time $\times$ EN type effect) on the King's Stool scores change (\%). As shown in the Fig. 2, the King's Stool scores (expressed by \% changes) in the low-FODMAP group were significantly and steadily reduced during the intervention compared with the moderate- or high-FODMAP groups, which were observed in all of the diarrhea subgroups (all of $P$-values $<0.05)$.
Alteration of anthropometric and biochemical parameters in response to EN treatment

Anthropometric and basic biochemical parameters according to EN treatment before and after the intervention are presented in Table 3 and Additional file 1: Table S2. After the intervention, BMI significantly increased in the low- and high-FODMAP groups ( $P<0.05$ for both), and a trend toward increasing was observed in the moderateFODMAP group $(P<0.10)$ (Table 3$)$. After the intervention, serum levels of uric acid were significantly reduced in the low-FODMAP group compared with the moderateand high-FODMAP groups $(P<0.05$; Table 3$)$. The liver function parameters serum AST $(P<0.01)$ and ALT $(P<$ $0.05)$ increased significantly after the intervention in the moderate-FODMAP group, and the kidney function parameter serum BUN was significantly increased in the high-FODMAP group $(P<0.05)$. However, all these values were within the normal range (Table 3). Other anthropometric parameters (e.g., weight, MAC, TSF, MAMC, and $\mathrm{BP}$ ) and basic biochemical parameters (e.g., $\mathrm{Hb}_{\mathrm{AlC}}, \mathrm{WBC}$, ALC, glucose, albumin, lipid profiles, and inflammatory 


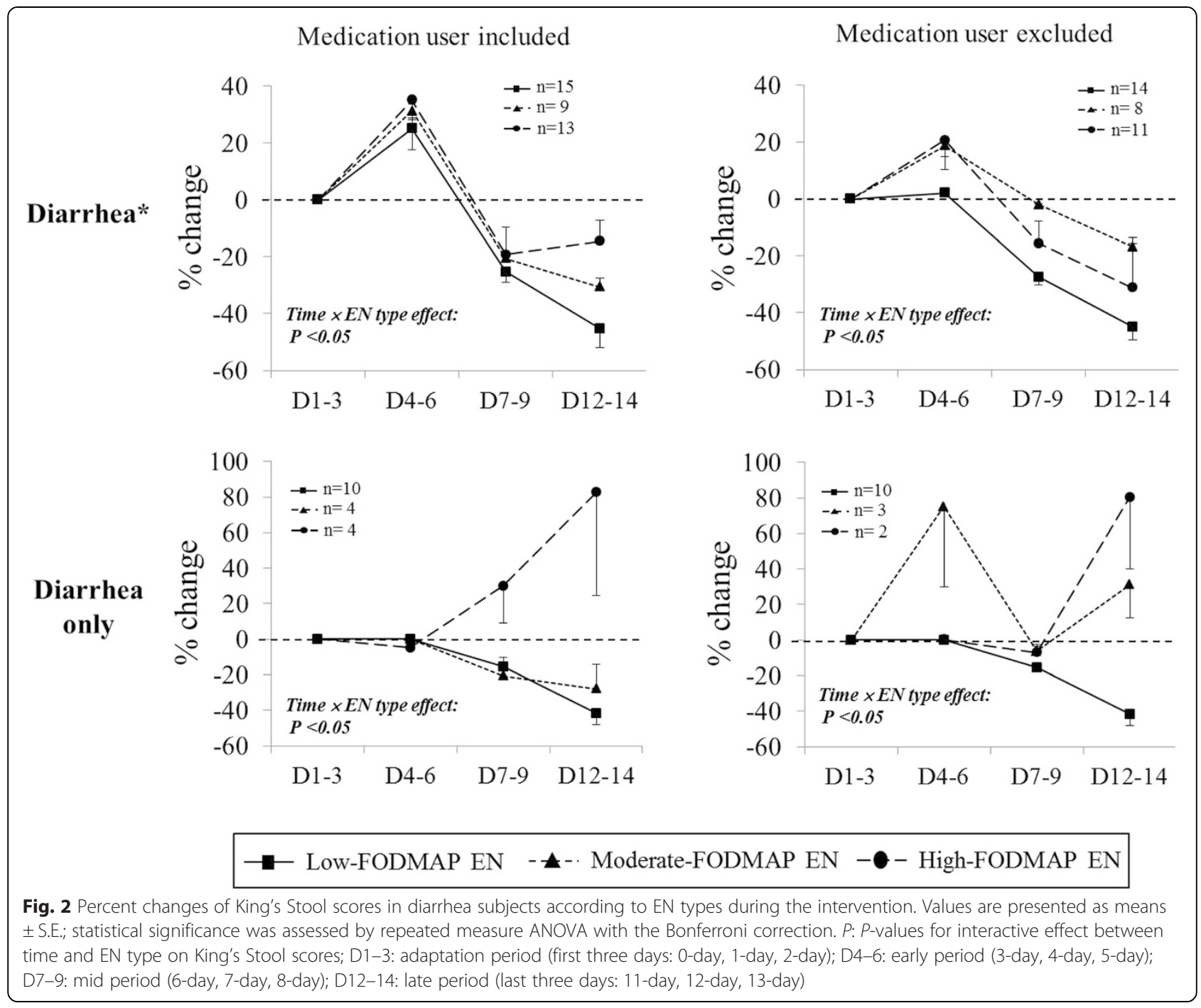

markers) did not significantly change over the course of the intervention in any of the three groups (Table 3 and Additional file 1: Table S2).

\section{Alteration of short-term nutritional status in response to EN treatment}

Serum albumin levels reflect nutritional status over the recent 20 days. As the intervention period in this study was only 14 days, serum prealbumin and transferrin, which reflect recent nutritional status (over the recent 2-3 days and 8-10 days, respectively), were also measured (Fig. 3). Serum levels of prealbumin increased significantly after the intervention in all three EN groups. By 3-day, serum prealbumin levels had significantly increased in the low- and high-FODMAP groups; of note, levels in the low-FODMAP group at 3-day had reached the levels at 14-day. By contrast, serum transferrin levels significantly increased after the intervention in the
moderate-FODMAP group, but not in the low- or highFODMAP groups.

\section{Nutritional status after the intervention, stratified by subject condition}

Study subjects were classified into five groups, according to their condition after the intervention (unimproved, normal-maintenance, diarrhea-improved, constipationimproved and recurrent D/C-improved: 1) unimproved ( $n=29$, no improvement from diarrhea or constipation), 2 ) normal maintenance ( $n=22$ without symptoms of diarrhea or constipation from the 23 subjects without symptoms before intervention), 3) diarrhea improved ( $n=8$ improvements from the 18 diarrhea-only subjects before the intervention), 4) constipation improved ( $n=13$ improvements from the 24 constipation-only subjects before the intervention) and 5) recurrent $\mathrm{D} / \mathrm{C}$ improved ( $n=12$ improvements from the 19 complex condition subjects before the intervention). Additional file 1: Table S3 
Table 3 Anthropometric measurements, blood cell counts, and liver and kidney function markers before and after the intervention

\begin{tabular}{|c|c|c|c|c|c|c|}
\hline & Low-FODMAP & & Moderate-FOD & $(n=28)$ & High-FODMAP & \\
\hline & Baseline & After & Baseline & After & Baseline & After \\
\hline$\overline{\text { Weight }(\mathrm{kg})^{\mathrm{a}}}$ & $55.4 \pm 2.37$ & $55.6 \pm 2.36$ & $56.7 \pm 2.61$ & $56.3 \pm 2.52$ & $55.9 \pm 2.48$ & $55.9 \pm 2.49$ \\
\hline BMI $\left(\mathrm{kg} / \mathrm{m}^{2}\right)^{\mathrm{a}}$ & $19.9 \pm 0.68$ & $21.3 \pm 0.90^{*}$ & $20.6 \pm 0.77$ & $22.2 \pm 1.07^{\dagger}$ & $20.4 \pm 0.75$ & $22.2 \pm 0.95^{*}$ \\
\hline MAC (cm) & $25.2 \pm 0.53$ & $25.5 \pm 0.58$ & $25.4 \pm 0.70$ & $25.2 \pm 0.77$ & $24.2 \pm 0.80$ & $24.8 \pm 0.66$ \\
\hline TSF (mm) & $11.0 \pm 0.89$ & $12.0 \pm 0.82$ & $12.2 \pm 1.04$ & $12.7 \pm 1.00$ & $10.1 \pm 0.88$ & $11.0 \pm 0.92^{\dagger}$ \\
\hline MAMC (cm) & $21.8 \pm 0.50$ & $21.7 \pm 0.56$ & $21.5 \pm 0.53$ & $21.2 \pm 0.66$ & $21.0 \pm 0.72$ & $21.3 \pm 0.61$ \\
\hline Systolic BP (mmHg) & $111.2 \pm 2.85$ & $110.8 \pm 2.46$ & $116.3 \pm 2.21$ & $116.8 \pm 2.36$ & $116.5 \pm 2.76$ & $117.6 \pm 3.49$ \\
\hline Diastolic BP (mmHg) & $74.2 \pm 2.52$ & $74.0 \pm 2.22$ & $73.5 \pm 1.52$ & $75.7 \pm 1.87$ & $75.0 \pm 1.89$ & $74.6 \pm 2.08$ \\
\hline HbA1c (\%) & $5.46 \pm 0.27$ & $5.47 \pm 0.34$ & $5.52 \pm 0.17$ & $5.43 \pm 0.18$ & $5.17 \pm 0.12$ & $5.24 \pm 0.13$ \\
\hline 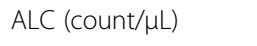 & $1957.7 \pm 132.1$ & $1858.1 \pm 83.2$ & $1791.2 \pm 123.1$ & $1763.2 \pm 121.2$ & $1769.2 \pm 137.2$ & $1790.0 \pm 147.5$ \\
\hline WBC $\left(\times 10^{6} / \mu \mathrm{L}\right)$ & $6.62 \pm 0.42$ & $7.17 \pm 0.48$ & $7.55 \pm 0.65$ & $7.12 \pm 0.53$ & $6.90 \pm 0.54$ & $7.84 \pm 0.59$ \\
\hline AST (U/L) & $20.8 \pm 1.11$ & $20.8 \pm 1.50$ & $20.3 \pm 1.25$ & $23.3 \pm 1.32^{* *}$ & $18.6 \pm 0.95$ & $17.8 \pm 0.89$ \\
\hline $\operatorname{ALT}(\mathrm{U} / \mathrm{L})$ & $21.8 \pm 2.87$ & $21.5 \pm 2.44$ & $16.3 \pm 1.29$ & $20.2 \pm 1.59^{*}$ & $14.3 \pm 1.25$ & $15.3 \pm 1.40$ \\
\hline Creatinine (mg/dL) & $0.67 \pm 0.05$ & $0.67 \pm 0.04$ & $0.70 \pm 0.07$ & $0.72 \pm 0.07$ & $0.62 \pm 0.04$ & $0.61 \pm 0.04$ \\
\hline BUN (mg/dL) & $14.1 \pm 1.64$ & $13.8 \pm 1.07$ & $13.9 \pm 1.40$ & $13.5 \pm 1.41$ & $11.7 \pm 0.96$ & $12.7 \pm 1.05^{*}$ \\
\hline Uric acid (mg/dL) & $4.48 \pm 0.27$ & $3.99 \pm 0.21^{*}$ & $5.10 \pm 0.33$ & $4.93 \pm 0.27$ & $4.74 \pm 0.29$ & $4.47 \pm 0.37$ \\
\hline
\end{tabular}

ALC absolute lymphocyte count, $A L T$ alanine aminotransferase, $A S T$ aspartate aminotransferase, $B M I$ body mass index, $B P$ blood pressure, $B U N$ blood urea nitrogen, $H b A 1 c$ glycated hemoglobin, MAC midarm circumference, MAMC midarm muscle circumference, TSF triceps skin fold thickness, WBC white blood cells

Values shown as means \pm S.E

${ }^{\text {a }} n=62$

$+p<0.1,{ }^{*} p<0.05,{ }^{* *} p<0.01,{ }^{* * *} p<0.001$. Differences in within-group means before and after the intervention were assessed using the paired $t$-test; there were no significant differences in the baseline values among the three groups

presents the percentage of the low-, moderate-, and highFODMAP EN consumption in each of subgroups: $75 \%$ of the diarrhea improved group consumed low-FODMAP EN formula. $38.5 \%$ and $46.2 \%$ of the recurrent $\mathrm{D} / \mathrm{C}$ improved group consumed the low- and moderateFODMAP EN formulas, respectively (Additional file 1: Table S3). As shown in Fig. 4, after the intervention, BMI significantly increased in the normal maintenance, the diarrhea improved, the constipation improved and the recurrent $\mathrm{D} / \mathrm{C}$ improved groups, but not in the unimproved group. TSF showed a trend toward increasing in the diarrhea-improved group, but it did not reach statistical significance. Serum prealbumin levels in the diarrheaimproved, the constipation improved and the recurrent D/C improved groups significantly increased at 14-day. Particularly, serum preablumin levels in the diarrhea improved and the recurrent $\mathrm{D} / \mathrm{C}$ improved groups were highly increased at 3-day and continued by 14-day. In the normal maintenance group, serum preablumin levels tended toward increasing at 14-day, but it did not reach statistical significance. Serum transferrin levels increased significantly in the diarrhea improved and the recurrent

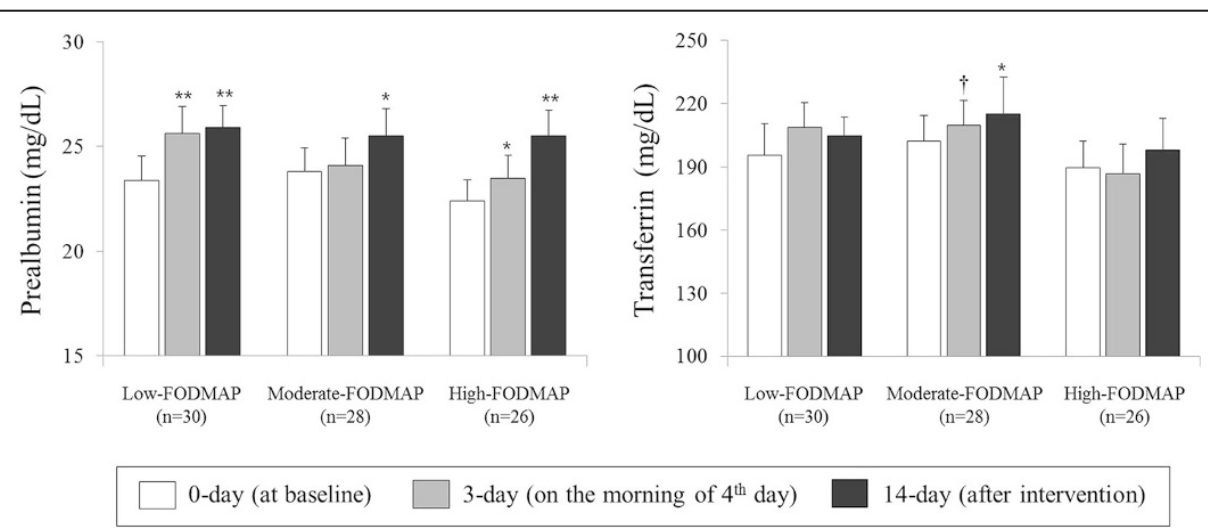

Fig. 3 Short-term nutritional status in response to EN treatment. Values presented as means \pm S.E.; statistical significance of within group differences was assessed by paired $t$-test. There were no significant differences in baseline values among the three groups. $† P<0.1,{ }^{*} P<$ $0.05,{ }^{* *} P<0.01$ compared with baseline values 


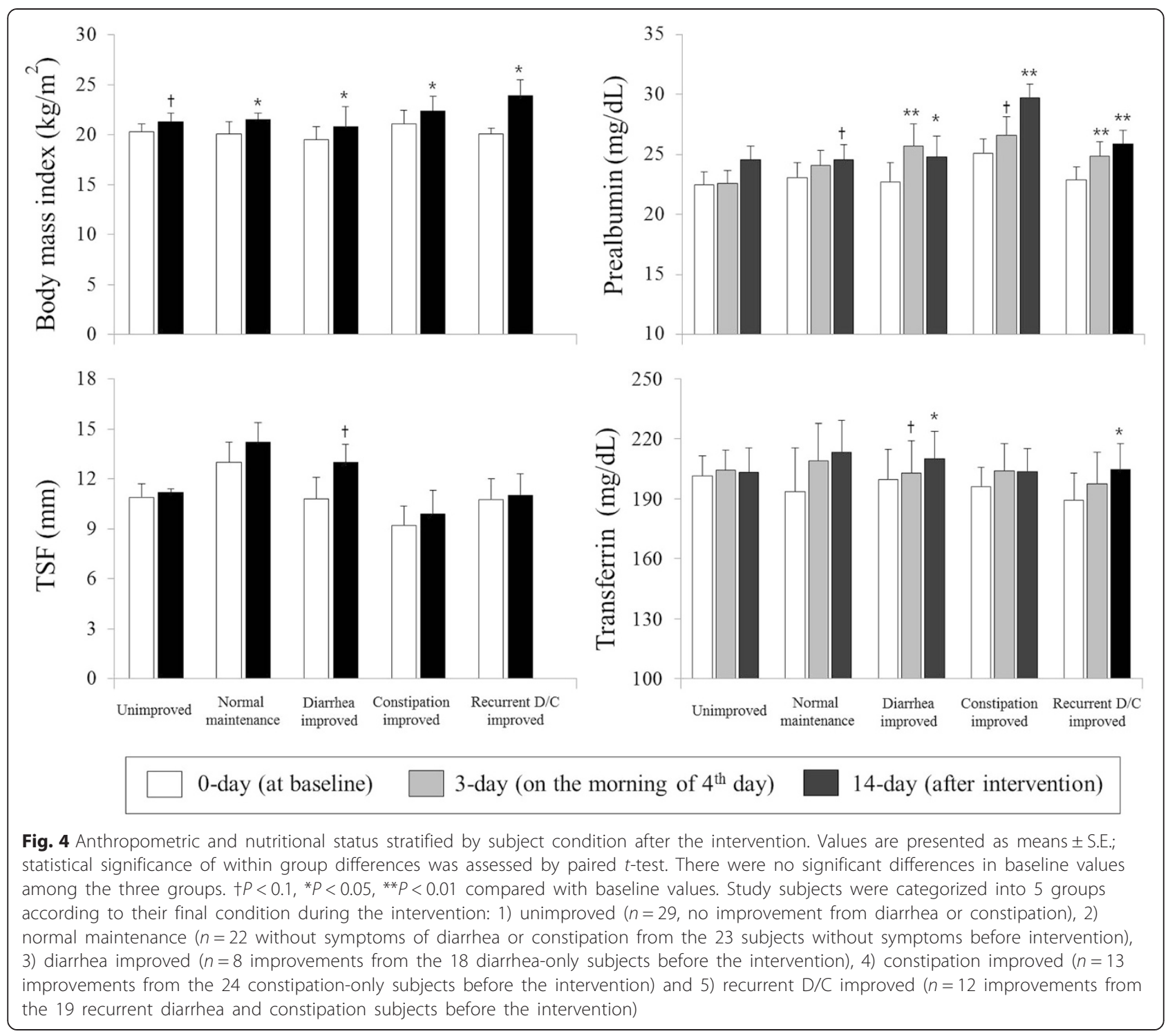

D/C improved groups at 14-day. By contrast, the unimproved group did not show any statistically significant changes in serum levels of prealbumin or transferrin.

\section{Discussion}

This randomized, multicenter, double-blind clinical trial shows for the first time that a low-FODMAP formula can promptly improve diarrhea and nutritional status in patients receiving EN. In this study, diarrhea in patients receiving low-FODMAP EN was significantly improved compared with those receiving moderate- or highFODMAP EN. King's Stool scores in patients with diarrhea in the low-FODMAP group were also significantly reduced than those in the other two groups. In addition, serum prealbumin, a marker of short-term nutritional status, promptly improved in patients receiving lowFODMAP EN, in contrast to the other groups. When subjects were classified by condition after the intervention, markers of short-term nutritional status (prealbumin and transferrin) were significantly improved in the gastrointestinal symptom-improved groups, particularly in the diarrhea-improved group. These results support the hypothesis that low-FODMAP formula may improve or reduce the likelihood of diarrhea in patients receiving EN, leading to an improvement in nutritional status helpful for prompt recovery from illness.

Diarrhea is one of the major gastrointestional problems frequently observed in hospitalized patients receiving EN [8-10]; it may cause nutritional imbalance and consequently delay recovery $[12,17]$. Many studies had tried to improve gastrointestinal problems, particularly diarrhea related to EN $[2,6,9,10,14,15,32]$, but the results have been inconsistent. Recent studies have reported an association between gastrointestinal symptoms 
and dietary FODMAPs. Restriction of dietary FODMAPs has been reported to improve gastrointestinal symptoms, including diarrhea, in a majority of patients with IBS and IBD [20-23]. Halmos et al. suggested in his retrospective study that receiving a lower-FODMAP formula may reduce the likelihood of diarrhea among several types of formulas [8]. In our present study, patients receiving a low-FODMAP EN formula showed greater improvements in diarrhea symptoms than those who received a moderate- or high-FODMAP formula (improvement in $60.0-73.3 \%$ of patients vs. $25.0-33.0 \%$ and 25.0-36.4\%, respectively). After the 14-day intervention, the low-FODMAP group also showed a much greater reduction in the King's Stool score compared with the other two groups. The mechanisms by why FODMAP restriction provides these benefits are assumed to be related to the molecular size and osmotic properties of FODMAPs [8, 33, 34]. FODMAPs are short-chain carbohydrates which have relatively smaller molecular size than non-short chain carbohydrates [8, 33, 34]. They are poorly absorbed in the small intestine and rapidly fermented by bacteria in the colon. Subsequent luminal distension may lead to secondary motility disturbance and diarrhea [23, 25]. Therefore, low-FODMAP formulas may result in less water retention within the lumen of the bowel and less gas in the intestine compared with moderate- or high-FODMAP formulas [8, 33, 34], thereby reducing gastrointestinal symptoms, particularly diarrhea.

In our study, compared with the other two groups, the low-FODMAP group showed significant improvements in serum prealbumin, a marker of short-term nutritional status at 3-day, which continued by 14-day, and in serum uric acid at 14-day. Additionally, patients with improved gastrointestinal symptoms after the intervention had greater BMIs than those who did not improve. Serum levels of prealbumin and transferrin which reflect recent nutritional status were also significantly improved in the improved gastrointestinal symptoms groups, particularly more in the diarrhea-improved group. It may be explained by that an improvement in diarrhea symptoms helps patients absorb sufficient amounts of nutrients to satisfy their nutritional requirements. These results from our randomized, double-blind, clinical trial provide the first intervention-based evidence that lowFODMAP formula improves diarrhea and nutritional status in patients receiving EN, potentially improving their nutritional status and ability to promptly recover from illness.

Our study has a few limitations. First, it is a randomized, double-blind, clinical trial where subjects were randomly assigned to one of three EN formulas. If each subject had consumed all three EN formulas via a crossover design, our conclusions would be more strongly supported. Second, long-term observation (i.e., for 2 to 6 months) may confirm whether the association between FODMAP-reduced EN feeding and improved diarrhea results in durable improvements to nutritional status and other metabolic parameters, shortening recovery time. Despite these limitations, this prospective intervention study shows that a low-FODMAP EN formula may improve diarrhea in patients receiving EN therapy, thereby improving nutritional status and potentially aiding prompt recovery from illness. Therefore, lowFODMAP EN formulas are expected to provide a useful therapeutic approach for patients with gastrointestinal symptoms, and particularly diarrhea, who are undergoing EN.

\section{Conclusions}

The results from our randomized, double-blind, clinical trial provide the first intervention-based evidence that low-FODMAP EN improves diarrhea and nutritional status in patients receiving EN, potentially improving their nutritional status and ability to promptly recover from illness. Therefore, low-FODMAP EN formulas are expected to provide a useful therapeutic approach for patients with diarrhea undergoing EN.

\section{Additional file}

Additional file 1: Figure S1. Improvement (\%) of constipation and recurrent diarrhea/constipation according to EN types during the intervention. Table S1. Baseline distribution of major gastrointestinal intolerance in the study subjects. Table S2. Biochemical markers of nutritional status, lipid profiles, and inflammation, before and after the intervention. Table S3. Proportion of the low-, moderate-, and high-FODMAP EN consumption according to the subjects' condition. (DOCX 488 kb)

\section{Abbreviations}

ALC: Absolute lymphocyte count; ALT: Alanine aminotransferase; AST: Aspartate aminotransferase; BMI: Body mass index; BP: Blood pressure; BUN: Blood urea nitrogen; EN: Enteral nutrition; FODMAPs: Fermentable oligosaccharides, disaccharides, monosaccharides, and polyols; Gl: Gastrointestinal; HbA1c: Glycated hemoglobin; HDL: High-density lipoprotein; hs-CRP: High-sensitivity C-reactive protein; IBD: Inflammatory bowel disease; IBS: Irritable bowel syndrome; IL-6: Interleukin-6; IL$\beta$ : interleukin-beta; LDL: Low-density lipoprotein; MAC: Midarm circumference; MAMC: Midarm muscle circumference; TSF: Triceps skin fold thickness; TNF-a: Tumor necrosis factor-alpha; WBC: White blood cells.

\section{Competing interests}

The authors declare that they have no competing interests.

\section{Authors' contributions}

OYK and JHL designed the research; OYK, SRY, JHL, JHL, and GYN conducted the research; $\mathrm{KHL}, \mathrm{YBL}$, and $\mathrm{GHJ}$ provided experimental EN formulas; OYK, SRY, and JHL analyzed the data; OYK and SRY wrote the draft; OYK contributed to the writing of the paper and had primary responsibility for final content. All the authors read, commented on, and contributed to the submitted manuscript.

\section{Acknowledgments}

We thank the research volunteers who participated in the studies and the medical staff from participating hospitals. This study was supported by the 
Basic Science Research Program through the National Research Foundation of Korea funded by the Ministry of Science, ICT and Future Planning (2013R1A1A3011535) and by Dr. Chung's Foods Co., Ltd. Dr. Chung's Foods Co., Ltd. had no influence over the study protocol or the analysis or interpretation of the results.

\section{Author details}

${ }^{1}$ Department of Food Science Nutrition, Dong-A University, Brain Busan 21 Project, Busan 604-714, Republic of Korea. ${ }^{2}$ Department of Rehabilitation Medicine, Dong-A University Hospital, Busan, South Korea. ${ }^{3}$ Central Research Institute, Dr. Chung's Foods Co., Ltd., Cheongju, Chungbuk, Republic of Korea.

Received: 8 July 2015 Accepted: 28 October 2015

Published online: 03 November 2015

\section{References}

1. Trabal J, Leyes P, Hervas S, Herrera M, de Tallo Forga M. Factors associated with nosocomial diarrhea in patients with enteral tube feeding. Nutr Hosp. 2008;23:500-4.

2. Luft VC, Beghetto MG, de Mello ED, Polanczyk CA. Role of enteral nutrition in the incidence of diarrhea among hospitalized adult patients. Nutrition. 2008;24:528-35.

3. Yang M, Chen PY, Gong ST, Lyman B, Geng LL, Liu LY, et al. Costeffectiveness analysis of an enteral nutrition protocol for children with common gastrointestinal diseases in china: good start but still a long way to go. J Parenter Enteral Nutr. 2014;38:72S-6.

4. McClave SA, Martindale RG, Vanek VW, Roberts P, Taylor B, Ochoa JB, et al. Guidelines for the provision and assessment of nutrition support therapy in the adult critically ill patient: Society of Critical Care Medicine (SCCM) and American Society for Parenteral and Enteral Nutrition (A.S.P.E.N.). J Parenter Enteral Nutr. 2009;33(3):277-316.

5. Gungabissoon U, Hacquoil K, Bains C, Irizarry M, Dukes G, Williamson R, et al. Prevalence, risk factors, clinical consequences, and treatment of enteral feed intolerance during critical illness. J Parenter Enteral Nutr. 2014. Epub ahead of print.

6. Gottschlich MM, Warden GD, Michel M, Havens P, Kopcha R, Jenkins M, et al. Diarrhea in tube-fed burn patients: incidence, etiology, nutritional impact, and prevention. J Parenter Enteral Nutr. 1988;12:338-45.

7. Staudacher HM, Whelan K, Irving PM, Lomer MC. Comparison of symptom response following advice for a diet low in fermentable carbohydrates (FODMAPs) versus standard dietary advice in patients with irritable bowel syndrome. J Hum Nutr Diet. 2011;24:487-95.

8. Halmos EP. Role of FODMAP content in enteral nutrition-associated diarrhea. J Gastroenterol Hepatology. 2013;28:25-8.

9. Halmos EP, Muir JG, Barrett JS, Deng M, Shepherd SJ, Gibson PR. Diarrhoea during enteral nutrition is predicted by the poorly absorbed short-chain carbohydrate (FODMAP) content of the formula. Aliment Pharmacol Ther. 2010;32:925-33.

10. Arevalo-Manso JJ, Martinez-Sanchez P, Juarez-Martin B, Fuentes B, Ruiz-Ares G Sanz-Cuesta BE, et al. Enteral tube feeding of patients with acute stroke: when does the risk of diarrhoea increase? Intern Med J. 2014;44:1199-204.

11. Cataldi-Betcher EL, Seltzer MH, Slocum BA, Jones KW. Complications occurring during enteral nutrition support: a prospective study. J Parenter Enteral Nutr. 1983;7:546-52.

12. DeMeo M, Kolli S, Keshavarzian A, Borton M, Al-Hosni M, Dyavanapalli M. Beneficial effect of a bile acid resin binder on enteral feeding induced diarrhea. Am J Gastroenterol. 1998;93:967-71.

13. Whelan K. Enteral-tube-feeding diarrhoea: manipulating the colonic microbiota with probiotics and prebiotics. Proc Nutr Soc. 2007;66:299-306.

14. Dial S, Alrasadi K, Manoukian C, Huang A, Menzies D. Risk of Clostridium difficile diarrhea among hospital inpatients prescribed proton pump inhibitors: cohort and case-control studies. CMAJ. 2004;171:33-8.

15. McFarland LV. Epidemiology of infectious and iatrogenic nosocomial diarrhea in a cohort of general medicine patients. Am J infect control. 1995;23:295-305.

16. Kyne L, Sougioultzis S, McFarland LV, Kelly CP. Underlying disease severity as a major risk factor for nosocomial Clostridium difficile diarrhea. Infect Control Hosp Epidemiol. 2002;23:653-9.
17. Lee JS, Auyeung TW. A comparison of two feeding methods in the alleviation of diarrhoea in older tube-fed patients: a randomised controlled trial. Age Ageing. 2003;32:338-93.

18. Bittencourt AF, Martins JR, Logullo L, Shiroma G, Horie L, Ortolani MC, et al. Constipation is more frequent than diarrhea in patients fed exclusively by enteral nutrition: results of an observational study. Nutr Clin Pract. 2012;27:533-9.

19. Barrett JS, Shepherd SJ, Gibson PR. Strategies to manage gastrointestinal symptoms complicating enteral feeding. J Parenter Enteral Nutr. 2009;33:21-6.

20. Halmos EP, Power VA, Shepherd SJ, Gibson PR, Muir JG. A diet low in FODMAPs reduces symptoms of irritable bowel syndrome. Gastroenterology. 2014;146:67-75.

21. Pedersen N, Vegh Z, Burisch J, Jensen L, Ankersen DV, Felding M, et al. Ehealth monitoring in irritable bowel syndrome patients treated with low fermentable oligo-, di-, mono-saccharides and polyols diet. World J Gastroenterol. 2014;20:6680-4.

22. de Roest RH, Dobbs BR, Chapman BA, Batman B, O'Brien LA, Leeper JA, et al. The low FODMAP diet improves gastrointestinal symptoms in patients with irritable bowel syndrome: a prospective study. Int J Clin Pract. 2013;67:895-903

23. Magge S, Lembo A. Low-FODMAP diet for treatment of irritable bowel syndrome. Gastroenterol Hepatol. 2012;8:739-45.

24. Staudacher HM, Irving PM, Lomer MC, Whelan K. Mechanisms and efficacy of dietary FODMAP restriction in IBS. Nat Rev Gastroenterol Hepatol. 2014;11:256-66.

25. Shepherd SJ, Lomer MC, Gibson PR. Short-chain carbohydrates and functional gastrointestinal disorders. Am J Gastroenterol. 2013;108:707-17.

26. Gearry RB, Irving PM, Barrett JS, Nathan DM, Shepherd SJ, Gibson PR. Reduction of dietary poorly absorbed short-chain carbohydrates (FODMAPs) improves abdominal symptoms in patients with inflammatory bowel disease-a pilot study. J Crohns Colitis. 2009;3:8-14.

27. Whelan K, Judd PA, Taylor MA. Assessment of fecal output in patients receiving enteral tube feeding: validation of a novel chart. Eur J Clin Nutr. 2004;58:1030-7.

28. Whelan K, Judd PA, Preedy VR, Taylor MA. Covert assessment of concurrent and construct validity of a chart to characterize fecal output and diarrhea in patients receiving enteral nutrition. J Parenter Enteral Nutr. 2008;32:160-8.

29. Frohmader TJ, Chaboyer WP, Robertson IK, Gowardman J. Decrease in frequency of liquid stool in Enterally Fed Critically III patients given the Multispecies probiotic VSL\#3: A pilot trial. Am J Crit Care. 2010;19:e1-11.

30. Kim OY, Lim HH, Lee MJ, Kim JY, Lee JH. Association of fatty acid composition in serum phospholipids with metabolic syndrome and arterial stiffness. Nutr Metab Cardiovas Dis. 2013;23:366-74

31. Song J, Kwon N, Lee MH, Koh Y, Lee JH, Kim OY. Association of serum phospholipid PUFAs with cardiometabolic risk: beneficial effect of DHA on the suppression of vascular proliferation/inflammation. Clin Biochem. 2014; $47: 361-8$

32. Thibault R, Graf S, Clerc A, Delieuvin N, Heidegger CP, Pichard C. Diarrhoea in the ICU: respective contribution of feeding and antibiotics. Crit Care. 2013;17:R153.

33. Whelan K, Schneider SM. Mechanisms, prevention, and management of diarrhea in enteral nutrition. Curr Opin Gastroenterol. 2011;27:152-9.

34. Barrett JS, Gearry RB, Muir JG, Irving PM, Rose R, Rosella O, et al. Dietary poorly absorbed, short-chain carbohydrates increase delivery of water and fermentable substrates to the proximal colon. Aliment Pharmacol Ther. 2010;31:874-82.

\section{Submit your next manuscript to BioMed Central and take full advantage of:}

- Convenient online submission

- Thorough peer review

- No space constraints or color figure charges

- Immediate publication on acceptance

- Inclusion in PubMed, CAS, Scopus and Google Scholar

- Research which is freely available for redistribution 